\title{
Raising Healthy Children: Packing a Peanut-Free Lunch ${ }^{1}$
}

\section{Sarah Schmidt, Karla Shelnutt, and Gail Kauwell ${ }^{2}$}

Peanut butter is a staple in the diets of many children, but it can cause serious problems for those with a peanut allergy. Knowing how to identify foods that contain peanuts, what to pack for school lunches, and how to reduce accidental contact with peanuts will help keep children with peanut allergies safe when they are away from home.

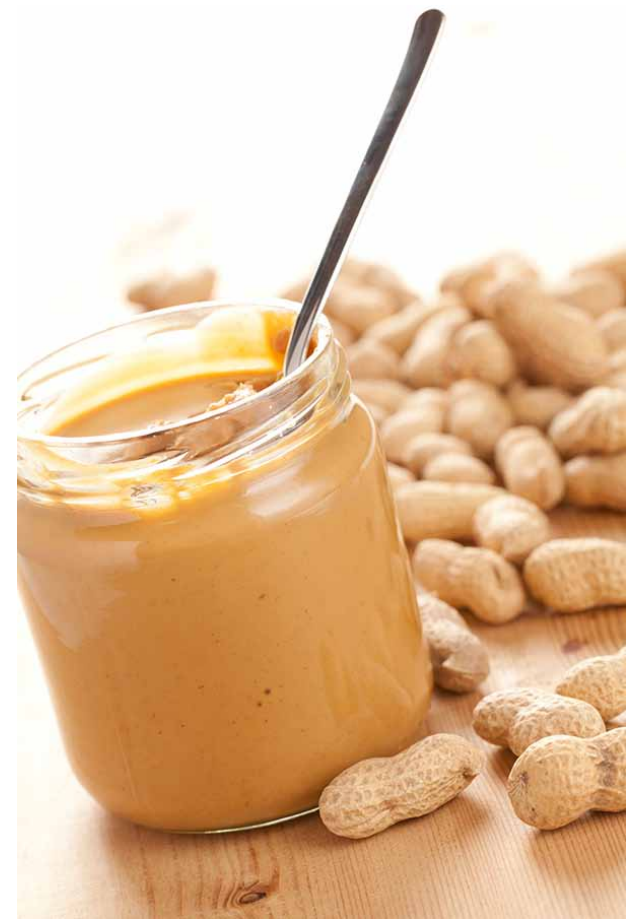

Figure 1. Peanut butter is known as a kid-friendly food, but it can be trouble for those with a peanut allergy.

Credits: iStockphoto

\section{Peanut Allergies What Is a Food Allergy?}

Food allergies occur when the body's immune system makes a mistake by reacting to a food that is usually harmless. Peanuts are a common cause of a food allergy in infants and children. Other foods that often cause allergies include eggs, milk, soy, shellfish, wheat, and tree nuts (such as walnuts). Children may outgrow some food allergies, but a peanut allergy is typically lifelong and can be life threatening (National Institute of Allergy and Infectious Diseases, 2010). In the United States, $4 \%-8 \%$ of children have food allergies (Stein, 2009). Of these, 1.4\% have peanut allergies (Sicherer, Muñoz-Furlong, Godbold, \& Sampson, 2010). For more information on food allergies, see the EDIS publication Raising Healthy Children: Food Allergies at http://edis.ifas.ufl.edu/fy1282.

\section{Symptoms}

Not every child with a peanut allergy reacts the same way, and the reaction produces symptoms that can range from mild to severe (Stein, 2009). Common signs include an itchy mouth, swelling of the lips and tongue, stomach cramps, diarrhea, vomiting, trouble breathing, dizziness, rash, and a drop in blood pressure (Centers for Disease Control and Prevention, 2012). In a severe reaction, the throat swells and the airways tighten, making it hard to breathe. This type of reaction occurs very quickly after eating or touching peanuts and can be deadly. Some children

1. This document is FCS80019, one of a series of the Department of Family, Youth and Community Sciences, Florida Cooperative Extension Service, Institute of Food and Agricultural Sciences, University of Florida. Original publication date February 2013. Please visit the EDIS website at http://edis. ifas.ufl.edu.

2. Sarah Schmidt, dietetic intern, Food Science and Human Nutrition Department; Karla Shelnutt, PhD, RD, assistant professor, Department of Family, Youth and Community Sciences; and Gail Kauwell, PhD, RD, LD/N, professor, Food Science and Human Nutrition Department; Florida Cooperative Extension Service, Institute of Food and Agricultural Sciences, University of Florida, Gainesville, FL 32611. 
have reactions just from inhaling particles from peanut flour or peanut cooking oil present in the air. Because of the possible danger, many children with a peanut allergy carry a medication (EpiPen ${ }^{\odot}$, epinephrine) that can be used to stop this reaction before it causes serious harm (The Food Allergy and Anaphylaxis Network, 2012). Teaching your child and the people who care for your child how to use the EpiPen ${ }^{\otimes}$ is critical to making sure your child recovers safely from an exposure to peanuts.

\section{Foods that Contain Peanuts}

Switching to a peanut-free diet can be tricky, but learning some basics about ingredients and processing can help reduce the chance of coming into contact with peanuts. Peanuts have many different names, including beer nuts, goobers, ground nuts, mixed nuts, nut pieces, nut meat, mandelonas, and monkey nuts (The Food Allergy and Anaphylaxis Network, 2011).

Even foods that do not have the word "peanut" or "nut" in the ingredient list can have peanuts in them. The Food and Drug Administration (FDA) requires that foods with any trace of peanuts be labeled in one of two ways. The label either needs to state the name of the allergen somewhere near the ingredients list: "contains peanuts"; or the ingredients list must state the source of the allergen: "oil (peanut)." Always read the food label to verify if a food contains peanuts. Never assume any food is peanut-free until you have checked the label (Mayo Clinic, 2012). Keep in mind that some foods are excused from the FDA labeling law. These include fresh fruits and vegetables; foods regulated by the USDA, like meats, poultry, or certain egg products; restaurant foods; and supplements. Non-food items, like medications, mouthwash, toothpaste, shampoo, and shaving cream, also are excluded.

Some common foods that often contain peanuts are listed below (The Food Allergy and Anaphylaxis Network, 2011):

- Asian food (dishes such as shrimp stir-fry and sweetand-sour chicken are often prepared with peanut sauce or peanut oil)

- Mexican food (such as enchiladas and beef sauces)

- Cookies, cakes

- Pastries

- Chocolate bars

- Candies
- Energy bars

- Ice cream, frozen yogurt

- Cereal and granola

- Breads topped with seeds and nuts

- Salad dressings

- Flavorings (natural and artificial)

- Chili

- Egg rolls

- Marzipan

Encourage your child to be cautious of these foods when served outside the home, such as at school parties and celebrations.

\section{Cross-Contamination}

Even if a food does not have peanuts in it, this does not mean it has not come into contact with peanuts. When preparing foods for children allergic to peanuts, avoid using the same utensils or surfaces where you had previously prepared peanut-containing foods. Traces of peanut not visible to the eye can remain on utensils and surfaces. This is similar to germs left behind on surfaces where food was prepared. The invisible traces of peanuts left behind could be transferred to the peanut-free food you are preparing for your child. Be sure to wash utensils and surfaces thoroughly with hot, soapy water and dry with a paper towel or clean dish cloth. If your child has a severe allergy, consider using separate kitchen equipment to prepare all peanut-free foods.

Cross-contamination is also an issue when purchasing food products. Many foods are made and processed in the same place as peanut-containing foods. Even though these foods do not include peanuts as an ingredient, they may contain traces of peanut that could cause an allergic reaction. The label of these foods may include the phrase "this food was processed in a factory that processes peanuts" or a similar statement, but this is not required. If you are worried a food may have been processed with peanuts and it is not indicated on the label, you can contact the manufacturer for more information or avoid giving the food to your child. 


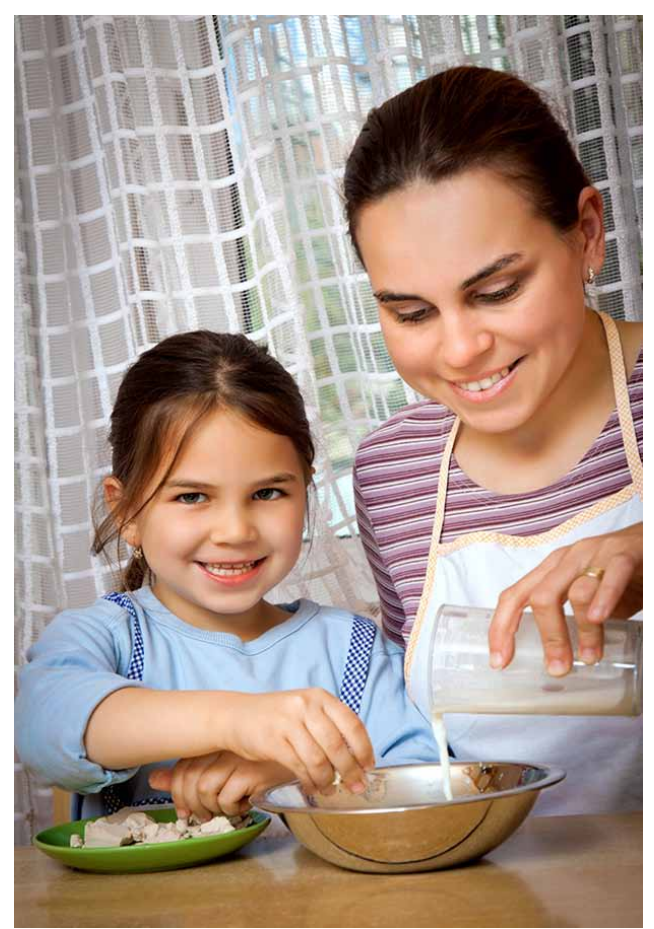

Figure 2. If your child has a peanut allergy, be aware that even peanutfree foods can come into contact with peanuts. Thoroughly wash any utensil or surface that might have touched peanuts, and always check food labels to see if a food was processed in a peanut-free facility. Credits: iStockphoto

\section{Peanut-Free Foods}

Even though many foods contain peanuts or traces of peanuts, there are still a lot of peanut-free foods. Fresh foods - produce, milk, fish, unprocessed whole grains (such as brown rice and popcorn) - are usually good choices.

\section{Packing a Safe and Nutritious Lunch}

Preparing a healthy, peanut-free lunch that includes kidfriendly foods is possible. Try these lunch ideas:

- Sandwiches - Instead of peanut butter and jelly, try sunbutter* and jelly. Sunbutter is made with sunflower seeds, and it is safe for kids with peanut or tree nut allergies. Ham or turkey sandwiches are always a hit. Use cookie cutters to make fun shapes, such as stars, flowers, or fish.

- Pasta - Add tomato or Alfredo sauce to bowtie, penne, angel hair, or your child's favorite pasta. Toss in some grilled zucchini or broccoli. Heat and pack in a thermal container. Many of these containers can keep food at a safe $140^{\circ} \mathrm{F}$ for up to four hours.
- Pack whole-grain crackers ${ }^{\star}$, cheese squares, sliced plum tomatoes, and pickle chips. Your child can assemble her own mini-sandwiches at lunch.

- Pack a whole-grain tortilla filled with corn-and-bean salsa, grilled chicken strips, green peppers, onions, and shredded cheddar cheese.

- Make egg salad using light mayonnaise, celery, garlic powder, and salt/pepper to taste. Add a thin layer to a bagel. Serve with a side of low-fat yogurt; pack fresh blueberries and blackberries for a fun yogurt topping.

- Pita dippers - Cut a whole-wheat pita into 8 slices. Add a container of pre-packaged hummus and black-bean dip for fun dipping. Serve with red bell pepper slices and fresh baby carrots.

- Cereal - Pack peanut-free cereal in a seal-tight container. Add a separate container of cinnamon, pumpkin seeds, and raisins ${ }^{\star}$ for your child to sprinkle on the cereal at school. Have your child add low-fat or fat-free milk from the cafeteria to his container of cereal.

- Kabobs - Sandwich on a stick! Put lightly toasted bread cubes $^{*}$, cheddar cheese cubes, ham (cut into 11/2-inch cubes), and apple slices onto a skewer. Serve with low-fat honey-mustard dressing for dipping. For a more Italian flavor, use mozzarella balls, grape tomatoes, olives, and basil, served with Italian dressing for dipping. The possibilities are endless!

- Trail mix - Help your child select his favorite ingredients to make a yummy trail mix. Add pretzels, raisins, baked cheese-flavored crackers, peanut-free granola, popcorn, and any other ingredient that your child loves and is safe to eat.

- Fresh fruits and vegetables and low-fat or fat-free milk are great choices to add to any lunch. Put an apple, banana, blueberries, carrots, celery sticks, or other fresh fruits or vegetables into your child's lunch, and have your child buy a carton of low-fat or fat-free milk at school for a complete meal.

${ }^{\star}$ Check to make sure these items are processed in a peanutfree facility.

School lunches are not guaranteed to be safe for your child. If you are interested in having your child buy school lunches, talk with the school's food services director about menus and ingredients to determine which foods are safe. 
The school may require a letter from your pediatrician stating that it is okay for your child to buy school-prepared lunch. The only way to make sure that your child's lunch is safe is to pack it at home. Packing a peanut-free lunch can be fun for you and your child. Just remember: Never assume any food is peanut-free. Check the label.

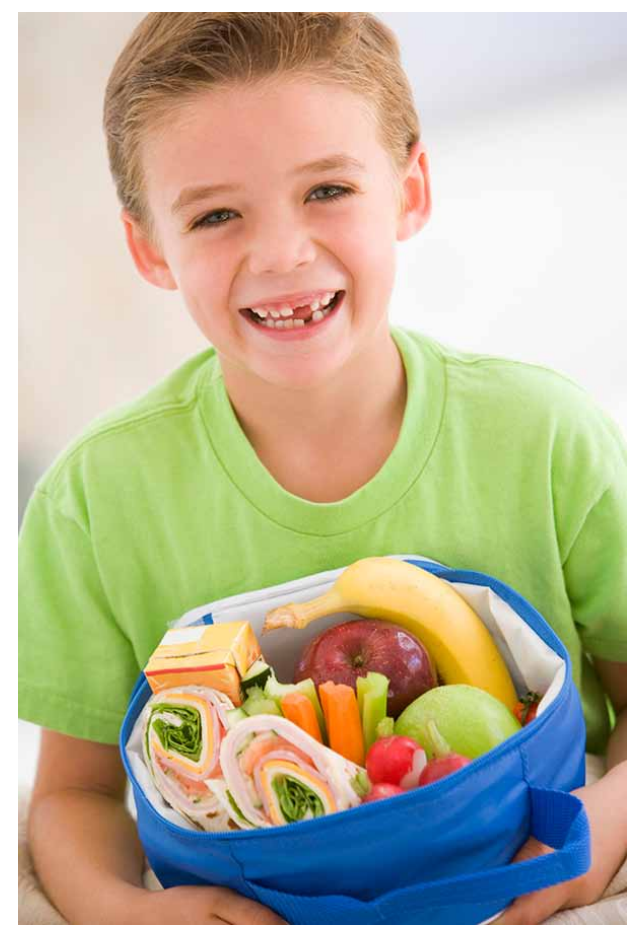

Figure 3. Packing a peanut-free lunch for your child can be fun and creative. Use the ideas in this publication and always include fresh fruits and vegetables.

Credits: iStockphoto

\section{Other Tips for Keeping Your Child Safe}

Along with preparing safe foods for your child, there are other steps you can take to reduce the chance of exposure to peanuts. Ask your child's teacher to tell you ahead of time when there will be a party in the classroom. Provide a peanut-free treat instead of other foods and make sure your child only eats the treat you provide. This way your child can enjoy the party and eat some food too. Giving your child a medical bracelet to wear is another good safety measure. Wearing a medical bracelet can alert others to your child's allergy and can ensure that your child receives proper treatment in an emergency.

\section{Summary}

Peanut allergies can be a serious health risk, so as a parent or caregiver you must provide safe, peanut-free foods to your child. Knowing what foods commonly contain peanuts and how to identify these foods using the food label can ease anxiety associated with preparing peanut-free foods. Training your child (and teachers, coaches, and other caretakers) about what foods to avoid is crucial to ensure your child is safe when away from home. Preparing a healthy, kid-friendly, peanut-free lunch is possible and can be fun for you and your child.

\section{Recommended Resources}

To get more information about allergies, healthy lifestyles, and nutrition, use the following websites and contact information.

UF/IFAS Extension (http://solutionsforyourlife.ufl. edu/families_and_consumers/health_and_nutrition/) - Provides open access to the EDIS website, the comprehensive, single-source repository of all current UF/IFAS peer-reviewed publications. Start here to explore Health and Nutrition topics in Florida. Learn more about specific nutrients and packing healthy lunches.

Mayo Clinic (http://www.mayoclinic.com/health/peanutallergy/DS00710) - This site contains valuable information about peanut allergies, including symptoms, causes, risk factors, complications, diagnosis, treatments, and coping.

The Food Allergy and Anaphylaxis Network (http://www. foodallergy.org/page/peanut-allergy) - This site has easyto-follow, practical advice about how to avoid sources of peanuts, commonly asked questions about peanut allergies, and recipes designed for people with one or more allergies.

\section{UF/IFAS Extension Family and Consumer Sciences} (FCS) Educator (look in the blue pages of your telephone book) - UF/IFAS Extension offices are listed online at http://solutionsforyourlife.ufl.edu/map.

Florida Dietetic Association - For referral to a registered dietitian $(\mathrm{RD})$ in your area, call the Florida Dietetic Association at (850) 386-8850 or check the yellow pages of your phone book.

\section{References}

Centers for Disease Control and Prevention. (2012). Food allergies in school. Retrieved from http://www.cdc.gov/ healthyyouth/foodallergies/

The Food Allergy and Anaphylaxis Network. (2011). How to read a label for a peanut-free diet. Retrieved from http:// www.foodallergy.org/files/HTRLsheet_2011.pdf 
The Food Allergy and Anaphylaxis Network. (2012).

Peanuts. Retrieved from http://www.foodallergy.org/page/

peanut-allergy

Mayo Clinic. (2012). Peanut allergy. Retrieved from http://

www.mayoclinic.com/health/peanut-allergy/DS00710

National Institute of Allergy and Infectious Diseases.

(2010). Food allergy: An overview. U.S. Department of

Health and Human Services. National Institutes of Health.

NIH Publication No. 11-5518. Retrieved from http://www.

niaid.nih.gov/topics/foodallergy/documents/foodallergy.

pdf

Sicherer, S. H., Muñoz-Furlong, A., Godbold, J. H., \&

Sampson, H. A. (2010). US prevalence of self-reported peanut, tree nut, and sesame allergy: 11-year follow-up. Journal of Allergy and Clinical Immunology 125 (6):1322-26.

Stein, K. (2009). Are food allergies on the rise, or is it misdiagnosis? JADA 109(11):1832-37. 